\title{
CONDITIONING OF A NEW GUN CAVITY TOWARDS 60 MV/m AT PITZ*
}

\author{
S. Lederer ${ }^{\dagger}$, G. Asova, J.W. Baehr, C. Boulware, H-J. Grabosch, M. Haenel, S. Khodyachykh, \\ S. Korepanov, M. Krasilnikov, B. Petrosyan, S. Rimjaem, T. Scholz, L. Staykov, \\ F. Stephan (DESY, Zeuthen, Germany) \\ K. Boyanov (INRNE, Sofia, Bulgaria) \\ D. Richter (BESSY GmbH, Berlin, Germany) \\ J. Roensch (University of Hamburg, Hamburg, Germany) \\ L. Hakobyan (YerPhI, Yerevan, Armenia) \\ P. Michelato, L. Monaco, C. Pagani, D. Sertore (INFN Milano - LASA, Segrate (MI), Italy)
}

\begin{abstract}
A new L-band photoelectron gun (prototype 3.2) has been installed for use with a $10 \mathrm{MW}$ klystron at the PhotoInjector Test facility in Zeuthen (PITZ). This gun has been conditioned starting in April 2007 up to a maximum gradient of about $60 \mathrm{MV} / \mathrm{m}$. The gun was tuned for resonance at $1.3 \mathrm{GHz}$, and stable operation has been achieved with 100 $\mu$ s RF pulses with a repetition rate of $10 \mathrm{~Hz}$. Over certain periods the gun has been conditioned at high gradient with RF pulses of $400 \mu \mathrm{s}$, and conditioning has to be continued at longer pulse lengths up to $700 \mu \mathrm{s}$. Maximum power in the gun has been achieved with a new RF waveguide phase shifter with a $2 \pi$ range. Dark current measurements have been performed with several cathodes, including uncoated molybdenum, electropolished uncoated molybdenum, and cesium telluride. Also, the first photoelectrons have been accelerated with the new gun cavity.
\end{abstract}

\section{INTRODUCTION}

Operation of a short wavelength free electron laser in the self-amplified spontaneous emission regime requires a high-quality electron beam. A necessary step toward achieving required emittance for the European XFEL project is to raise the accelerating gradient in the gun cavity from $42-45 \mathrm{MV} / \mathrm{m}$ up to $60 \mathrm{MV} / \mathrm{m}[1]$. A new RF gun has been recently installed at PITZ and has been conditioned for short RF pulses up to maximum output power of a $10 \mathrm{MW}$ klystron. The RF gun consists of a 1.5 cell copper cavity operated at $1.3 \mathrm{GHz}$ standing wave in the $\pi$ mode. The cathode system is able to insert one of several cathodes. Molybdenum cathodes have been used to condition the gun cavity and cesium telluride photocathodes for producing photoelectrons. The gun cavity is surrounded by the main and bucking solenoids for beam focusing and compensating spacecharge-induced growth of the projected emittance. A detailed description of PITZ can be found in [2]. In this paper we present the conditioning history of the

\footnotetext{
* This work has partly been supported by the European Community, contracts RII3-CT-2004-506008 and 011935, and by the 'Impuls- und Vernetzungsfonds' of the Helmholtz Association, contract VH-FZ-005.

† sven.lederer@desy.de
}

new gun as well as dark current measurements with several cathodes.

\section{CONDITIONING HISTORY}

After tuning, the gun was cleaned including ultrasonic cleaning and high pressure rinsing with ultrapure water. Afterwards the cavity was treated under clean room conditions to avoid contamination with particles. Conditioning of the new gun with a $10 \mathrm{MW}$ klystron began on April 10th, 2007. The klystron has two waveguides and the sum forward power exceeds that which can be transmitted through a single RF vacuum window, so the power is transmitted using two waveguide arms. The RF power is combined close to the gun using a T-combiner. The relative phase of the RF in the two arms is adjusted with a waveguide phase shifter in one of the arms. The waveguide phase shifter used in the beginning of the conditioning process for gun 3.2 (Figure 1a) had a maximum phase adjustment of $90 \mathrm{deg}$ by changing the electrical length of one waveguide.

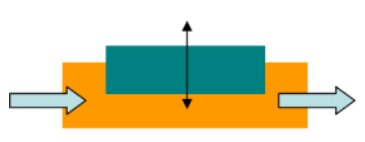

a)

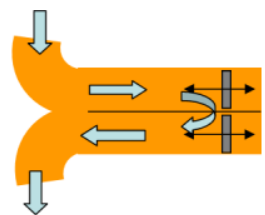

b)
Figure 1: Schematic designs for a) the old and b) the new phase shifter, black arrows show the mechanical movement in the phase shifter, and the cyan arrows represent the RF power flow

This phase shifter was replaced with a new one (Figure 1b) which allows the phase offset to be tuned over $2 \pi$. As schematically shown in Figure 1, the old phase shifter changes the electrical length of one waveguide to adjust the phase, by changing the volume in the waveguide, and so the $\mathrm{H}$-field. The new phase shifter design does not require reducing the distance between the waveguide walls, which reduces the probability of sparks inside the phase shifter at high RF power. Using the new waveguide phase shifter, the gun forward power presently exceeds $6 \mathrm{MW}$, with reflected 
power from the gun less than $300 \mathrm{~kW}$. The accelerating gradient at the cathode in the cavity is given by

$$
E_{0}=K_{0} \sqrt{P}
$$

where $P$ is the RF power in the gun and

$$
K_{0} \approx 2.36 \times 10^{4} \frac{\mathrm{V}}{\mathrm{m} \sqrt{\mathrm{W}}} .
$$

The progress of the conditioning is shown in Figure 2. Progress of the maximum gradient is made by various periods of conditioning by ramping up the RF power under the control of an automatic process. This program can reset the interlocks from vacuum sensors, temperature sensors, and photomultipliers that occur constantly as the maximum RF power (and therefore maximum gradient) in the gun is increased. After resetting the interlocks the ACP increases the RF power depending on certain RF power thresholds [3]. After conditioning to a given gradient without an external magnetic field, the gun is reconditioned up to the same gradient with varying fields provided by the main and bucking solenoids. The solenoid magnetic fields are scanned over the full range to vary the dark current distribution within the gun to clean the surface by electron bombardment.

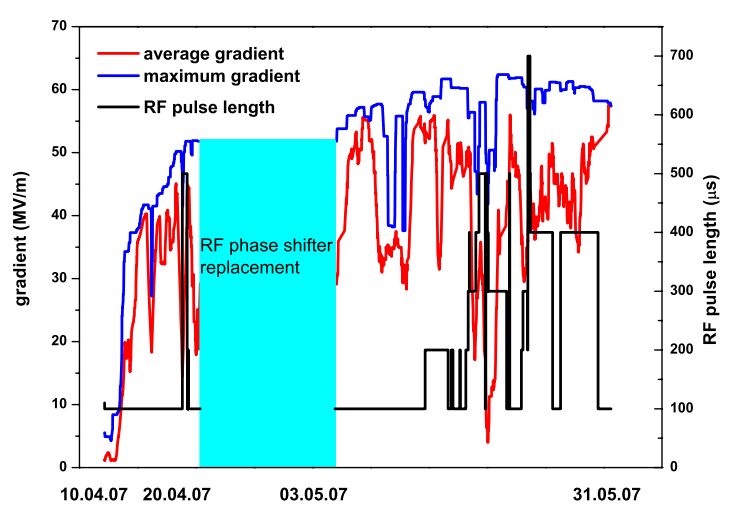

Figure 2: Conditioning history for gun 3.2 at PITZ. Average gradient (red line) represents the average accelerating gradient over a four-hour period, and maximum gradient (blue line) is the highest gradient achieved in the same fourhour window. The RF pulse length (black line) is plotted on the right axis.

In the beginning of the conditioning, the main reasons for interlocks are vacuum related. At high power and long RF-pulses photomultiplier interlocks and temperature interlocks on the inner conductor in the directional coaxial power coupler (partially heated by the high dark current) are identified as main reasons for slowing down the conditioning process.

\section{DARK CURRENT EVOLUTION}

Due to high RF fields at the surface of the gun cavity, field emission of electrons produces a large amount of dark current, which can be further accelerated. This results in bad vacuum conditions, which is a critical issue for $\mathrm{Cs}_{2} \mathrm{Te}$ photocathode operation. These extra electrons interfere with the detection of the photoemitted beam and excess dark current also causes several other problems for the accelerator not directly related to the photocurrent. Because it has a much higher duty cycle $(1.3 \mathrm{GHz}$ repetition rate) than the photoemitted beam (1 MHz repetition rate), dark current can dominate radiation production in the tunnel. Radiation in the PITZ tunnel damages many components, including diagnostics cameras, position switches for movable devices, and some kinds of water flow sensors. Deposited dark current also raises the temperature of the vacuum chamber wall at the impact point. Extreme increases in temperature can damage the chamber, but even moderate increases result in vacuum problems and can complicate the temperature regulation required to keep the gun in resonance. The dark current consists primarily of field emitted electrons from the cathode region and the surrounding gun area. To clarify this we compare the rise of the average current with electric field in the gun to that predicted from the Fowler-Nordheim theory for metallic surfaces [4]. The average current by field emission during a sinusoidally varying electric field pulse is given by

$$
I=K_{1} A\left(\beta E_{0}\right)^{\frac{5}{2}} \exp \left[\frac{K_{2}}{\beta E_{0}}\right]
$$

where $I$ is the average current during the pulse, $A$ is the effective emitting area, $\beta$ is the local electric field enhancement due to surface roughness, $E_{0}$ is the maximum macroscopic electric field, and $K_{1}$ and $K_{2}$ are coefficients which depend on the workfunction of the emitter [5]. A sample data set using a molybdenum cathode is shown in Figure 3. The increase in dark current with accelerating gradient is well-characterized by Equation 3. The effective emitting area is $10^{-8} \mathrm{~m}^{2}$ and effective field enhancement around 300 .

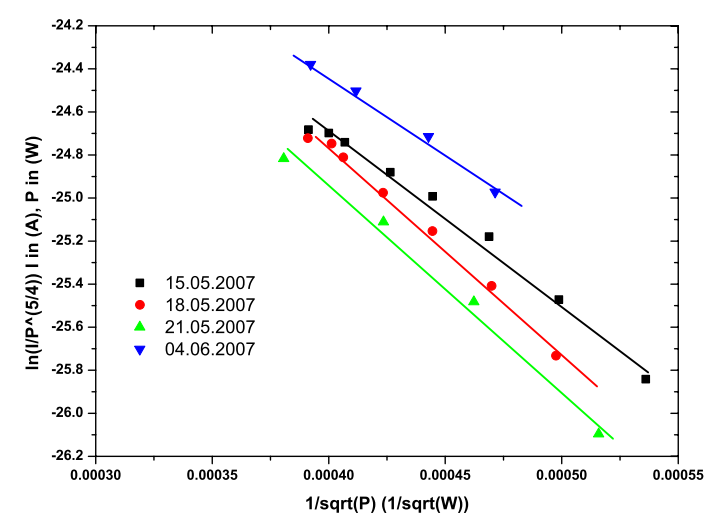

Figure 3: Dark current data for molybdenum cathode \#31.4 used in gun 3.2, with fits according to Equation 3

In Figure 4 maximum dark currents for different cathodes vs. gun power are presented. The maximum dark 
current is the highest current measured as function of the solenoid magnetic fields. The front surface of the plugs of all cathodes except \#55.3 have been optically polished by an automatic technique [6]. The uncoated Mo cathode \#32.2 shows highest values for dark current. This cathode was used during the beginning of the conditioning process. After a certain time cathode \#31.4 (same type as \#32.2) was used for conditioning, and a decrease of the dark current by more than a factor of two can be seen in Figure 4 . The lowest amount of dark current was found using the electropolished uncoated molybdenum cathode \#55.3. On this cathode not only the front surface, but the whole cathode was electropolished. Both cesium telluride cathodes produce dark currents comparable to the standard polished Mo cathodes. The relatively small values for the electropolished cathode show promise for a reduction in dark current by such cathode treatment.

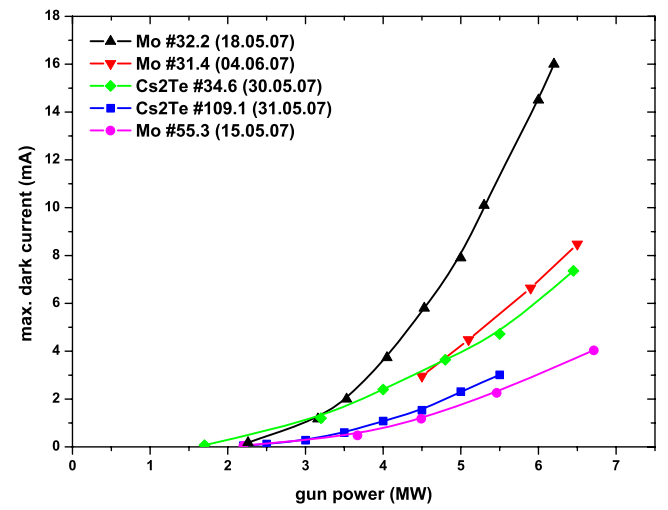

Figure 4: Dark current vs. gun power for different cathodes

A comparison with gun 3.1 [7] shows even for the electropolished cathode that the dark current is roughly a factor of two higher for gun 3.2. After the mounting of the cleaned gun the cavity had to be vented because of vacuum leaks in two consequent installed coaxial RF couplers, while for gun 3.1 this was not necessary. This could be one reason for the high dark current level of the present gun.

Besides the fact that the dark current follows the predictions from Fowler and Nordheim, Figure 3 clearly shows the dark current trend over time for this cathode. From the first data taken on 15.05.2007 the dark current decreases with time, which shows the conditioning process. In contrast, the dark current measured on 04.06.2007 is higher than before. This behavior can be explained by a damaging of the cathode surface during the conditioning. In Figure 5 photographs from cathode \#32.2 and \#31.4 after usage for conditioning are shown. On the surface of both cathodes one can see damage over the complete area. These damaged sites serve as field emitters themselves so that the dark current increases even with ongoing conditioning.
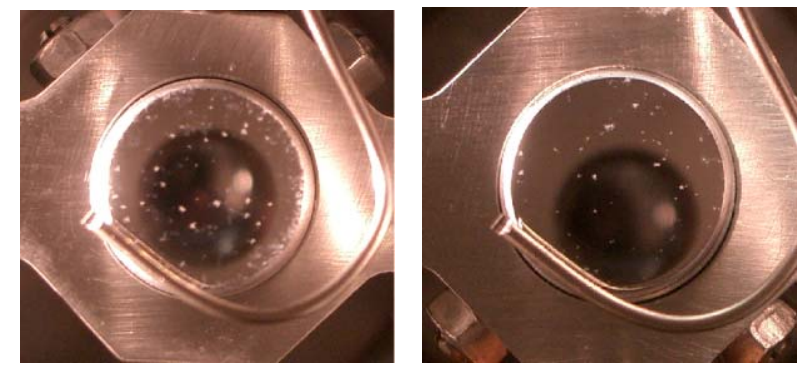

Figure 5: Damaged Molybdenum cathodes \#32.2 (left) and \#31.4 (right)

\section{CONCLUSIONS}

The new L-band gun 3.2 at PITZ has been conditioned to about $60 \mathrm{MV} / \mathrm{m}$ accelerating gradient at the cathode for short RF pulses up to $100 \mu$ s with $10 \mathrm{~Hz}$ repetition rate. This high gradient is a required design point for the European XFEL. At this gradient, the increased dark current causes increased radiation in the tunnel, leading to damage of various accelerator components. Cathodes damaged during the conditioning process (possibly induced by sparking) are strong sources of dark current, while electropolished molybdenum plugs show promise for reduced dark current. A solution to the problem of high dark current becomes more critical as the conditioning of the new gun will proceed to longer RF pulses. Further attempts to reduce the dark current are cathodes where not only the front surface, but also the edge is polished as well as dry-ice cleaning of the cavity.

\section{REFERENCES}

[1] M. Altarelli, et al. (eds.), "The Technical Design Report of the European XFEL", Chapter 4: XFEL accelerator. DESY 2006-097, July 2006, p. 80.

[2] A. Oppelt, et al., "Status of the PITZ Facility Upgrade", Proceedings of the LINAC2006, Knoxville, TN, USA, 21-25 August 2006

[3] A. Bohnet, et al., "Conditioning of the RF gun at the photo injector test facility at DESY Zeuthen", EPAC2002, La Villette-Paris, France, June 2002.

[4] R.H. Fowler and L. Nordheim, Proc. Roy. Soc. (London) A 119, 626 (1928).

[5] J.W. Wang, "RF Properties of Periodic Accelerating Structures for Linear Colliders", SLAC Report 339, 1989, p. 105.

[6] D. Sertore et al., "Review of the production process of the TTF and PITZ photocathodes", PAC05, Knoxville, Tennessee, USA, 2005.

[7] A. Oppelt, et al., "Tuning, Conditioning and Dark Current Measurements of a New Gun Cavity at PITZ", FEL06, Berlin, Germany, August - September 2006. 